rev.relac.int.estrateg.segur.10(1):225-243,2015

\title{
EL CAMINO DE CHILE ANTE NACIONES UNIDAS EN EL MARCO DE LA GUERRA DE IRAK: UNA MIRADA Y UN ACTUAR*
}

\author{
Erna Ulloa Castillo*
}

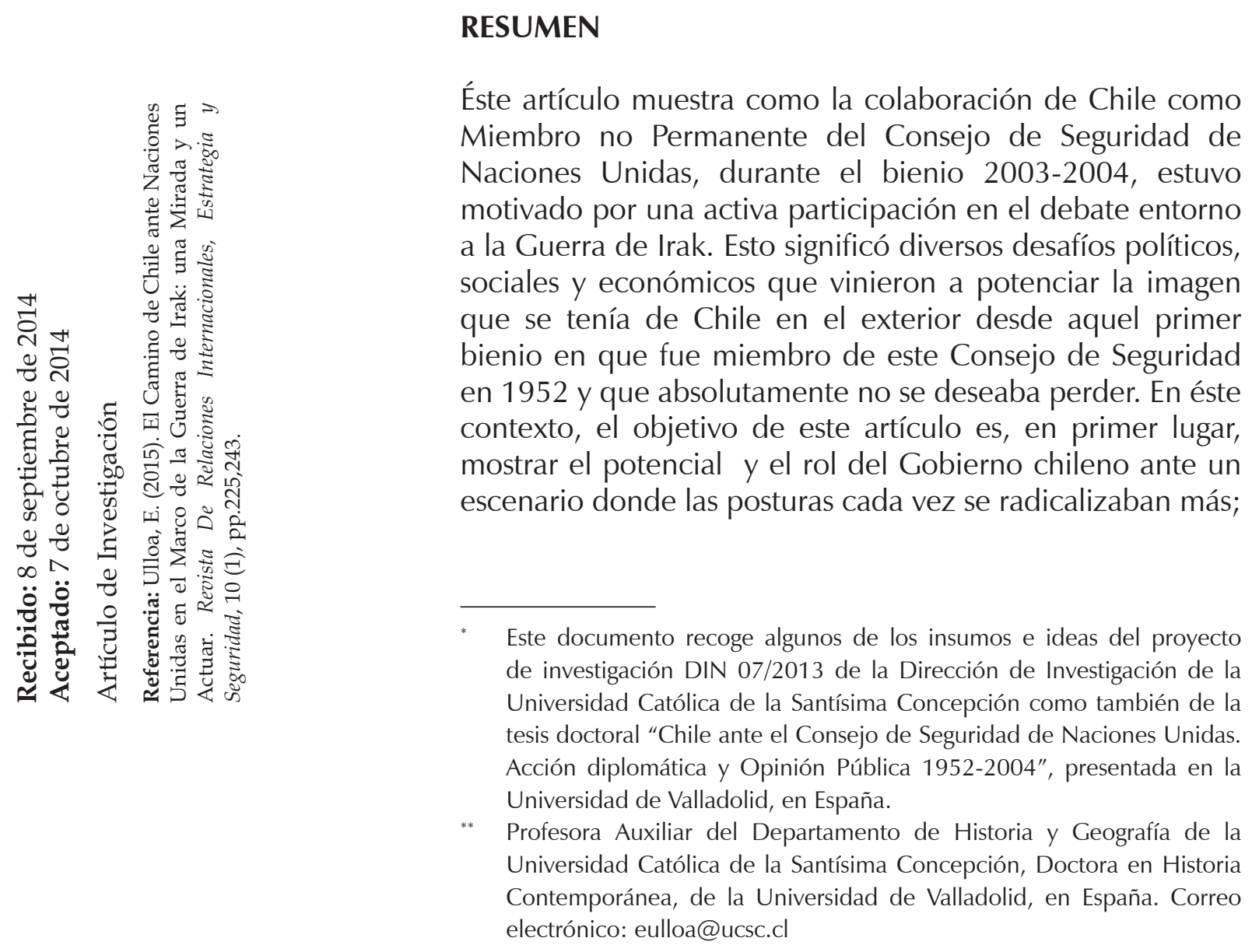


y en segundo lugar, analizar cómo la multilateralidad fue el mecanismo a seguir ante lo que lo Chile calificó como una guerra elegida más que una guerra necesaria en relación a unos de los mayores conflictos bélicos y humanitarios de inicios del siglo XXI. Para ello se analizaron fuentes primarias del Ministerio de Relaciones Exteriores de Chile y fuentes contrapuestas de prensa local como son los diarios El Mercurio y La Nación.

Palabras clave: Naciones Unidas, Consejo de Seguridad, Cooperación, Política Exterior, Multilateralidad.

\title{
THE WAY OF CHILE TO THE UNITED NATIONS FRAMEWORK FOR THE IRAQ WAR: A LOOK AND ACT
}

\begin{abstract}
This article shows how the collaboration of Chile as Not permanent Member of the Security Council of United Nations, during the period of two years 2003-2004, was motivated by an active participation in the debate about the War of Iraq. This meant diverse political, social and economic challenges that came to promote the image that had Chile in the exterior from that first period of two years in which he was a member of this Security Council in 1952 and which, absolutely one did not want to lose. In this context, the aim of this article is, first, to underline the potential and the role of the Chilean Government before a scene where the positions every time were becoming more radical more; and secondly, to analyze how the multilateralism was the mechanism to continuing before what Chile qualified as a war chosen more than a necessary war in relation to some of the major warlike and humanitarian conflicts of beginnings of the 21st century. For it, there were analyzed primary sources of the State Department of Chile and opposite sources of local press, The Mercury and The Nation newspaper.
\end{abstract}

Keywords: United Nations, Security Council, Cooperation, Foreign Policy, Multilateralism.

\section{O CAMINHO DE CHILE ANTE NAÇÕES UNIDAS NO MARCO DA GUERRA DE IRAQUE: UMA MIRADA E UM ATUAR}

\section{RESUMO}

O presente artigo mostra como a colaboração do Chile como Membro não Permanente do Conselho de Segurança de Nações Unidas, durante o biênio 2003-2004, esteve motivada por uma ativa participação no debate meio à Guerra do Iraque. Isto significou diversos desafios políticos, sociais e econômicos que vieram a potenciar a imagem que se tinha do Chile no exterior desde aquele primeiro biênio em que foi membro deste Conselho de Segurança em 1952 e que, absolutamente não se desejava perder. Neste contexto, o objetivo deste artigo é, 
em primeiro lugar, sublinhar o potencial e o papel do governo chileno ante um palco onde as posturas a cada vez se radicalizavam mais; e em segundo lugar, analisar como a multilateralidad foi o mecanismo a seguir ante o que o Chile qualificou como uma guerra eleita mais que uma guerra necessária em relação a uns dos maiores conflitos bélicos e humanitários de inícios do século XXI. Para isso se analisou fontes primárias do Ministério de Relações Exteriores do Chile e fontes contrapostas de imprensa local como são os diários O Mercurio e a Nação.

Palavras-chave: Nações Unidas, Conselho de Segurança, Cooperação, Política Externa, Multilateralismo.

\section{INTRODUCCIÓN}

Chile se enfrenta actualmente a un importante desafío e incertidumbre bajo el gobierno de la presidenta Michelle Bachelet, ya que a partir de enero del 2014, el país ha vuelto a ser elegido como Miembro no Permanente del Consejo de Seguridad para el bienio 2014-2015, en instantes que la búsqueda de acuerdos de paz no se vislumbran ante escenarios tan complejos como Siria o la Franja de Gaza.

La posibilidad de alianzas políticas entre los miembros de este organismo para adherir a resoluciones oficiales se ven de pronto absolutamente difusas; frente a ello, es innegable no recordar un episodio tan decisivo para la historia reciente de Chile cuando las presiones internacionales para poner un alto al fuego en Irak, se hicieron sentir en instantes que el país cumplía su tercer bienio al interior de este Consejo de Seguridad (2003-2004) . De allí que al analizar y recoger aquellos momentos surjan varios interrogantes: ¿̇uál fue la gran lección que trajo consigo este conflicto para Chile?, ¿̇cuál fue el impacto y el papel que desempeñó la prensa chilena ante la opinión pública?, ¿qué principios guiaron la política exterior chilena?

Al responder a estos interrogantes, se aportará al conocimiento de la historia de las relaciones internacionales de Chile, ya que hasta ahora existe un importante vacío sobre esta temática. Las investigaciones en torno a la participación de este país como Miembro no Permanente del Consejo de Seguridad, no ha sido un área mayormente abordado por los historiadores o cientistas políticos, quienes principalmente se han sumergido en el estudio de la política exterior, sin considerar las dinámicas sujetas a los bienios al interior de este organismo. Por lo tanto, el presente artículo será un aporte que llenará un vació y tributará a la historia del tiempo reciente de Chile, dejando abierto el camino para futuras investigaciones.

El presente documento, en primer lugar, abordará las medidas iniciales que asumió Chile frente a la situación de Irak, subrayando la importancia de la gestión diplomática para mantener

1. Chile ha sido parte del Consejo de Seguridad en los periodos: 1952-1953; 1961-1962; 1996-1997; 2004-2005; 2014-2015. 
la autonomía del país y unirse como una sola voz para expresar su posición a la agenda norteamericana en relación a la invasión de Irak.

Luego, se analizará la situación de las denominadas armas de destrucción masiva y la búsqueda de éstas por parte de la misión de especialistas enviados por Naciones Unidas. En este sentido, Chile abrigará la idea del tiempo, para que Irak demostrara colaboración ante las acusaciones de tener en su territorio, material armamentístico que no sólo afectaría a Medio Oriente, sino que también alimentaría la idea de terrorismo.

El tercer punto, analiza la gestión chilena como actor del concierto internacional frente a la decisión de invadir territorio iraquí. De allí, que se dé cuenta de una iniciativa del gobierno del presidente Ricardo Lagos, para asegurar el desarme de Irak por vías pacíficas, por medio de una fórmula centrada en 5 puntos claves que buscaban ser una vía alterna a la noción de guerra.

Finalmente, las conclusiones no solamente dan cuenta de los interrogantes planteados, sino que también permiten conocer el impacto que significaba estar ad portas de la firma de un Tratado de Libre Comercio con EEUU, en días en que este país requería del voto chileno a favor para llevar a cabo la invasión a Irak. Por lo tanto, el camino de Chile al interior del Consejo de Seguridad estuvo marcado por importantes desafíos, pero también por un aprendizaje en torno a cómo manejar la diplomacia y la política exterior frente a un organismo como la ONU.

De allí la importancia que reviste el estudiar y analizar los archivos del Ministerio de Relaciones Exteriores de Chile, que se transforman no sólo en material inédito, sino que también en una rica fuente de información. En donde también estudiar la prensa por medio de un análisis de contenido, permite llenar esos vacíos que la documentación oficial de pronto presenta.

\section{UNA GUERRA QUE INICIA UN NUEVO SIGLO: IRAK, CHILE Y EL CONSEJO DE SEGURIDAD DE LA ONU}

Si algo nos demostró el conflicto de Irak, es que a pesar de los cuestionamientos y críticas hacia la ONU, la necesidad de un organismo que permitiera una articulación del sistema internacional resultaba ser crucial para alcanzar la tan anhelada paz y seguridad internacional, propósito rector desde el mismo momento del nacimiento del organismo (Pereira y Martínez, 2001).

Ahora bien, ello no quiere decir que las discrepancias y diferencias al interior de éste, no se hicieran presentes, más aún cuando a cada minuto la idea de la presencia de armas de destrucción masiva podrían afectar no sólo a los países directamente relacionados con el conflicto, pues las consecuencias de un evento bélico se saben cuándo inician pero no cuando terminan. Asimismo y sumado a esto, la decidida postura de la dictadura de Saddam Hussein, dejaba de manifiesto un ambiente internacional denso y complejo (Pérez, 1998). 
Bajo este escenario, hay que considerar también el gran temor que hubo en torno a los efectos del terrorismo, particularmente sobre la población civil, sobre todo si consideramos la sofisticada capacidad de manipulación mediática e intoxicación informativa que estos grupos como dice Francisco Veiga (2009) llevan a cabo.

Por lo tanto, y a todas luces, surge la imperiosa necesidad de estrechar alianzas y en este sentido, Naciones Unidas debe responder como un agente de la seguridad internacional para la legítima defensa: "the fact that no judical machinery exists to hold the united nations responsible should not be allowed to obscure the fact that the united nations remains responsible for its internationally wrongful acts" (Talmon, 2007:32). La cual debe estar marcada no sólo por la independencia de la política exterior de cada nación, sino más profundo aún, velar por el propio derecho a la vida de sus ciudadanos (Remiro, 2003).

En este sentido, es importante subrayar que el Gobierno del presidente Lagos Escobar, durante 2000-2006 estableció desde un inicio del tercer bienio al interior del Consejo de Seguridad, que la Delegación chilena apostada en Nueva York cumpliera con dos objetivos claves que vendrían a sustentar el trabajo del equipo diplomático al interior de este Consejo. En primer lugar, conocer la postura de cada país respecto al conflicto de Irak y, en segundo lugar, solicitar a cada Delegación que elaborara un listado de temas de interés en común para llevarlo a la mesa del Consejo para su consiguiente plan de ejecución (Quezada, 2010).

El primer punto, resultó ser decisivo para las acciones que se llevaron a cabo posteriormente, puesto que era imperioso no tan solo para los miembros de Naciones Unidas, sino también para la propia opinión pública, conocer la visión de cada uno de los 15 miembros del Consejo de Seguridad frente al conflicto. Al respecto, desde el grupo latinoamericano, Colombia antes de terminar su periodo y cederle su puesto a Chile, había declarado que era una necesidad compartida el que Irak cumpliera con las resoluciones de la ONU, tal cual lo había manifestado la Canciller Carolina Barco².

Asimismo, Alemania que también se integraba por aquellos días al Consejo de Seguridad de mano del embajador Hans Heinrich, daba a conocer su más absoluta oposición a una guerra con Saddam Hussein, por lo tanto, ¿cuál era el camino que seguiría Chile? La respuesta, resulta ser muy gráfica ya que marcó a la política exterior del gobierno de Lagos, no tan sólo frente a este gran conflicto, sino que fue un verdadero faro que guió toda su administración frente a estos temas. Con ello nos referimos al respeto al orden jurídico internacional apegado a la propia carta constitutiva de la ONU y a la vez, investigar minuciosamente si se hallaban aquellas armas

2. Entrevista de Ministra de Relaciones Exteriores de Chile Soledad Alvear con Canciller de Colombia, Sra. Carolina Barco Isakson. Oficio № 100, Ministerio de Relaciones Exteriores, Gobierno de Chile, Misión Chile-ONU, 15 de septiembre, 2002. 
de destrucción masiva. Puesto que de ser así, se debía proceder a su eliminación inmediata de acuerdo a lo que estableciera el grupo de inspectores de este organismo encomendado para aquella labor, la Comisión de la ONU de Vigilancia, Verificación e Inspección (UNMOVIC).

Hay que entender que esta segunda guerra de Irak representó para Chile un importante desafío ya que ser parte del Consejo de Seguridad, era una ocasión que permitiría asentar la imagen país hacia el exterior (Arístegui, 2003). Asimismo y como lo sostiene el historiador chileno Joaquín Fermandois (2004), éstos espacios son apetecidos por la diplomacia y los internacionalistas chilenos. De allí entonces que la actuación a desempeñar fuera decisiva y clara, pero también sumamente cuidadosa más aún, cuando se estaba ad portas de la firma de un Tratado de Libre Comercio (TLC) con EEUU que venía gestándose desde el gobierno del presidente Eduardo Frei Ruíz-Tagle3 (Vargo, 2004; Agüero, 2005).

En este sentido, el gran temor era que trabajo de años podía evaporarse en cualquier minuto desterrando los beneficios que este acuerdo bilateral iba a traer no solo al pueblo chileno en términos económicos, sino también estratégicos y políticos para la propia administración de Lagos. Esto por cuanto, de firmar el TLC, sería gran triunfo a su gestión pensando sobre todo que el PIB podría aumentar en casi dos puntos. Pero paralelamente, ya se escuchaban voces como la del representante de Comercio de EEUU, Robert Zoellick, que "frente a la postura del presidente chileno existía una decepción de su gobierno, por no apoyar al ataque directo a Irak", a pesar de ello, Chile siguió manteniendo un lenguaje de apoyo a las acciones del equipo de inspectores de la ONU (Fazio, 2004, p.18).

\section{UN MOTIVO INQUIETANTE: ARMAS DE DESTRUCCIÓN MASIVA}

En la medida que un proceso de conflicto internacional se va desplegando, un rol importante es el que desarrolla la prensa y cómo impacta ésta con sus informaciones a la población civil y en esto la tranquilidad o inquietud que pueden transmitir resulta ser absolutamente crucial (Gomis, 1991).

Bajo este contexto, debemos plantear que los medios de prensa escrita chilenos marcaron una agenda informativa muy amplia sobre lo que estaba ocurriendo en Medio Oriente y de paso, presionaban al gobierno para que éste se manifestara. Fue el caso de inicios del 2003, cuando aquel temor global de la frase armas de destrucción masiva se enfrentaba ante lo que publicaban los diarios chilenos El Mercurio y La Nación: "Ios inspectores de armas de la

3. Debemos indicar al respecto, que fueron más de 12 años de negociación, ya que desde el Gobierno del Presidente Eduardo Frei Ruíz Tagle en 1994 se comenzó a trabajar en la iniciativa de negociaciones sobre un Acuerdo de Libre Comercio, a través de una modalidad bilateral, puesto que era tarea prioritaria para los intereses económicos de Chile. 
ONU encontraron ayer 11 ojivas vacías de 112 milímetros, que eventualmente pueden ser cargadas con armamento químico, en un almacén militar en Irak" (17 de enero, 2003, p.A-4.; 17 de enero, 2003, p.14). Si bien estas eran armas obsoletas, no habían sido ni informadas ni declaradas al grupo de inspectores de la UNMOVIC, dirigidos por Hans Blix, de todas formas el temor se hizo presente.

La actitud del Gobierno chileno ante lo que estaba viendo, podríamos graficarla en una palabra en la cual creía firmemente: tiempo. Esto, por cuanto para Chile como lo hemos indicado anteriormente, lo trascendental era apegarse al orden jurídico y en este sentido, las Resoluciones 1441 y $1284^{4}$, daban respuesta a esta noción que Chile planteaba. En este sentido, antes de invadir Irak para el presidente Lagos y su equipo diplomático, era importante dar el espacio suficiente para que este país colaborara llevando a cabo el desarme solicitado por el Consejo de Seguridad. Pero en contrapartida, EEUU se oponía por considerar que el tiempo se había agotado y que la actitud de Hussein se oponía al Business as usual: "Estaba claro que Washington no quería ningún subterfugio que le diera más tiempo a Saddam" (Muñoz, 2005, p.41).

Frente a estos acontecimientos que inundaban la agenda del Consejo de Seguridad, la prensa local chilena debatía sobre el accionar del país: "El gobierno de Chile no descarta aportar fuerzas militares a una operación de imposición de la paz en el Golfo Pérsico, siempre que sea la ONU quien realice la petición" (La Nación, 21 de enero 2003, p.14). Una iniciativa que por cierto no resulta para nada sorpresiva, sino todo lo contrario, puesto que la propia trayectoria chilena a lo largo de los cuatro bienios como Miembro no Permanente del Consejo de Seguridad, han dejado en evidencia un apego constante al derecho internacional y a los estatutos establecidos en la Carta Fundacional de la ONU (Llanos, 1996).

De allí entonces, que esto explique el que no se cuestionara lo que establecieran las diversas resoluciones oficiales, como también el considerar un posible aporte de fuerzas armadas para apoyar el contingente militar de paz de la ONU, más conocidos como los cascos azules. En este sentido, el actuar chileno debe ser visto y comprendido en el marco de una operación de mantenimiento de la paz, por lo tanto, alejado de todo favoritismo o compromiso hacia una nación determinada, por lo menos en esta ocasión.

4. Frente a ambas Resoluciones el gobierno norteamericano comunicó directamente a Chile cuál era la posición que ellos tenían al respecto. Así fue como el Primer Secretario de Asuntos Políticos de la Embajada de Estados Unidos en Chile, Tom Mittnmach, informó a la Cancillería chilena sus puntos de vistas centrados en dos ejes: a) Irak debía cumplir las todas las obligaciones impuestas por ambas resoluciones, b) no se podía pensar en el desarrollo de algunas de estas disposiciones si al 27 de enero de 2003 no había cumplido con la resolución 1441 y c) Irak no ha cooperado con lo cual si se le concedía mayor tiempo como establecía la resolución 1284 era caer en punto cero. Ver en: Mensaje Oficial 014, REF.: Posición EE.UU. respecto resolución sobre Irak, Ministerio de Relaciones Exteriores, Gobierno de Chile, Dirección de Política Multilateral, Departamento de Naciones Unidas 22 enero, 2003. 
Si bien, una opción era dar tiempo suficiente para alcanzar el desarme por el camino de la paz, también Chile tenía claro que éste tampoco podría ser indefinido (Muñoz, 2005). Por lo tanto, el punto medio entre ambas posturas, era operar de acuerdo a los principios del multilateralismo, ello explica entonces que en cada momento que le era posible, el gobierno chileno manifestara que se debían esperar los informes del equipo de especialistas que inspeccionaban la existencia de aquellas armas de destrucción masiva en Irak. Por consiguiente, el llamado de Lagos estuvo fuertemente marcado en tomar una decisión, sólo una vez que todos los miembros del Consejo de Seguridad tuviesen en las manos dichos informes: "La actitud chilena va a ser la de honrar su responsabilidad, privilegiar justamente las decisiones del Consejo de Seguridad y que sea la diplomacia multilateral la que opere" (La Nación, 23 de enero, 2003, p.4).

En tal sentido, el equipo diplomático chileno y como uno de los representantes del grupo latinoamericano, debía imperiosamente responder a la confianza que habían depositado sus pares regionales sobre ellos, en términos no sólo de la responsabilidad que recaía sobre sus hombros al ocupar un sitial dentro del grupo de los quince del Consejo de Seguridad, sino que también debía, por sobre todo, utilizar y promover los mecanismos de cooperación y consulta multilaterales, que resultaban ser los ejes axiales sobre los cuales se constituía la visión de lo que debía ser este organismo y el medio bajo el cual llevar adelante el tan anhelado desarme ${ }^{5}$.

Si bien, tanto Lagos como su delegación diplomática, tenían claridad en cómo debía trazarse el camino en pos del encuentro de la paz, la realidad al parecer hacía estrellar y diluir todo intento incipiente de esperanzas, por cuanto al correr de los días la inminente guerra se transformaba en realidad cuando: "Estados Unidos ya habría ordenado a sus tropas desplegadas en el Golfo Pérsico estar listas para un eventual enfrentamiento armado contra el régimen de Bagdad a partir del 10 de febrero", como lo informaba el diario árabe Al-Hayat, haciendo eco de ello también la prensa chilena (La Nación, 29 de enero, 2003, p.15). Lo llamativo de esta publicación árabe es que no estaba ni firmada ni mucho menos corroborada por fuentes oficiales, a pesar de ello fue publicada causando un revuelo mundial.

Ahora, si bien es cierto, que tanto el tiempo como la multilateralidad vinieron a ser ese mecanismo para aportar a una quizás ilusa o esquiva ilusión de paz en el corto plazo, desde la vereda de un país al sur del mundo como Chile surgía a su vez, una interrogante que cada vez tomaba más fuerza: "iqué sucedía con el papel y responsabilidad que debían cumplir los cinco Miembros Permanentes del Consejo de Seguridad?" (Ulloa, 2013:542). Pues, la ausencia de consensos y una acción en conjunto de partes de éstos, evidenció que se puso sobre la espalda una carga muy pesada a pequeños y alejados países de los grandes centros de poder como eran los 5 grandes. Al respecto, son elocuentes las declaraciones de la Delegación chilena a la prensa:

5. Mensaje Oficial № 026, Ministerio de Relaciones Exteriores, Gobierno de Chile, Dirección de Política Bilateral, 4 de febrero de 2003. 
"No es correcto que la solución del problema se esté dejando en manos de los miembros no permanentes, mientras Estados Unidos, Francia, Gran Bretaña, Rusia y China no hacen ningún esfuerzo para aproximar sus puntos de vista" (El Mercurio, 28 de febrero, 2003, p.1).

Paralelamente a la situación de Irak, se pusieron sobre el tapete, una vez más, dos problemáticas que hasta el día de hoy parecen no encontrar un acuerdo definitivo y sentencioso, nos referimos a ampliar el número de miembros no Permanentes del Consejo de Seguridad y regular el uso constante y reiterativo del veto por parte de los 5 Permanentes (Palomares y García, 1999). De allí, que resulte imprescindible ampliar la mirada a esta situación, puesto que "no hay que olvidar que este ya no es el mundo de Guerra Fría para seguir obrando de la misma forma cuando nació este organismo en 1945" (Ulloa, 2013, p.2).

Ahora bien, lo interesante de todo este debate y sobre el camino que debía seguir Chile ante las presiones que se hicieron presentes no sólo por parte de EEUU, sino que también de Reino Unido, llevó al gobierno de Lagos una importante lección en términos de cómo plantear los temas de política exterior ante la opinión pública. La estrategia chilena fue de recoger las opiniones de los más diversos sectores políticos y sociales del país, ello explica entonces que el presidente invitara al Palacio de La Moneda a todos los partidos políticos para que expresaran sus posturas respecto a Irak: "porque en estos temas todos los chilenos tienen algo que decir, no es un tema ni de gobierno ni de oposición, es un tema de país" (El Mercurio, 28 de febrero, 2003, p.1).

De esta manera quedaba en evidencia que para Lagos y su equipo diplomático, los asuntos de relaciones exteriores eran un canal de primer orden, en donde resultaba crucial hacer partícipe a los más diversos sectores de la política interna del país. En este sentido el paso de los años ha demostrado que sus sucesores, tanto el primer periodo de la presidenta Michelle Bachelet como el periodo del presidente Sebastián Piñera, comprendieron no sólo la lección, sino que también la trascendencia de hacer partícipe, al más variado espectro político y social: "Chile está entre los países que mayor grado de cumplimiento ha dado a estas metas en el mundo y que tienen que ver con reducir la pobreza, crear empleos, mejorar las oportunidades, eliminar los elementos de discriminación y dar oportunidades para que todos los ciudadanos de nuestro país puedan desarrollarse en plenitud" (Publimetro, 2013) .

De esta manera se logra que una postura ante Naciones Unidas, no deba responder al gobierno que esté de turno, sino ser una medida y actitud que trascienda en el tiempo para asentarse como una política exterior con sentido ciudadano (Milet, 2004; Mintz y De Rouen, 2010).

6. Esta cita se enmarca en las declaraciones del Presidente Sebastián Piñera en lo que fue la candidatura de Chile para integrar el Consejo de Seguridad en su quinto bienio. Edición online del diario electrónico Publimetro, 24 de septiembre de 2013.http://www.publimetro.cl/nota/politico/pinera-chile-va-muy-bien-encaminado-al-consejo-deseguridad-de-la-onu/xIQmix!|CsLVXgksboGM/ 
Bajo este escenario también se debe reconocer, por otro lado, que si bien los informes que entregaba la UNMOVIC poseían prácticamente una fuerza de ley para Chile, también le era gravitante la falta de cooperación que estaba demostrando Irak, en cuanto a dar conocer qué materiales poseía o bien cómo destruyó a aquellos con los cuales ya no contaba, incluyendo en esto la incertidumbre si se estaban desmantelando los misiles Al Samoud II7.

Así visto, el escenario claramente deja en evidencia profundas contradicciones: "Así el poder ha pasado a manos de quienes carecen de fuerza y se ha creado una situación dominada por la incertidumbre. La incertidumbre y la impredecibilidad como elementos que pueden transformase en un arma peligrosa en toda situación internacional" (Nicolson, 2010, p.237). Y en este sentido, debemos reconocer que es francamente imposible llegar a buen puerto cuando se obra sobre la base de una seudo multilateralidad anteponiendo los intereses personales.

Al respecto, un elemento que vino a tensionar aún más el papel de aquellos Miembros no Permanentes del Consejo de Seguridad, vino de mano del temor ante la falta de consensos y sobre todo, de la ausencia de dirección de los denominados cinco grandes. De allí que se planteara una tercera vía para conseguir el tan anhelado desarme pacífico de Irak. Esta opción planteada en conjunto por México y Canadá, pese a que éste último país no formaba parte del Consejo de Seguridad, consideraba imponer a Bagdad una serie de tareas concretas de desarme, las cuales tendrían plazos fijos y rígidos a cumplir.

De alguna manera, la tercera vía se vinculó directamente con aquellos países que fueron denominados como el grupo del miedo o bien los indecisos (U-6), dentro de los cuales se contaba a México, Guinea, Pakistán, Angola, Camerún y Chile, en términos que no deseaban invadir Irak sin pruebas, pero que tampoco podían esperar per se, a que éste demostrara resultados de ello. Cabe recordar que el Consejo de Seguridad estaba dividido entre los miembros que deseaban llevar a cabo la invasión como EE. UU., Gran Bretaña, España -principalmente- y los que estaban a favor de una prórroga de los inspectores por un tiempo indefinido como Francia, Alemania, Rusia y China con el apoyo de Siria, principalmente ${ }^{8}$.

Frente a la división, era imperioso que Koffi Annan, pusiera la voz de consenso ante tal la situación, lo que en su calidad de Secretario General resultaba esperable, más aún, si visualizamos que el Consejo de Seguridad fue considerado desde sus inicios como una especie de gabinete de acción tras el flagelo de dos guerras mundiales (Nicolson, 2010). Por lo tanto, sólo era viable y además con carácter de urgente, la unidad de sus miembros para salvarlos de un quiebre irremediable, aunque a decir verdad, desde el mismo momento de su conformación hasta el

7. Estos misiles se enmarcaron en el programa de armamento que impulsó Irak tras la Guerra del Golfo Pérsico en 1991.

8. Oficio № 097, Ref.: Proyecto resolución Sirio Consejo de Seguridad, Ministerio de Relaciones Exteriores, Gobierno de Chile, Misión Chile-ONU, 17 de abril, 2003. 
día de hoy, pareciera que siempre el Consejo de Seguridad y la ONU, están al borde de las constantes críticas sobre su gestión.

En concreto, no podemos dejar de reconocer que para Chile todo este escenario de discrepancias y desacuerdos sobre cómo operar respecto a Irak, evidenciaba que los cimientos que había presentado el Consejo de Seguridad durante el bienio anterior en que fue parte de éste (1996-1997 $)$, una vez más corroboraba la fragilidad sobre la cual se actuaba. Ante la indecisión del grupo del miedo, el diario londinense The Observer (2 de marzo, 2003) denunció en sus páginas (y que fue recogido por toda la prensa mundial) que EE. UU. estaba realizando un seguimiento de miembros indecisos para averiguar su postura sobre el conflicto iraquí. Consultada la Canciller chilena Soledad Alvear, por esta denuncia, fue enfática en señalar que estaban averiguando dicha situación: "Hemos instruido a nuestro Embajador en el Reino Unido para que recabe datos sobre la veracidad de las informaciones" (La Nación, 7 de marzo, 2003, p.12). Cierto o no, lo concreto de este hecho fue la presión que se estaba realizando y lo complejo que le resultaba a Chile toda esta situación, por cuanto si bien aparecía como un país estable, democrático y con una postura clara, no dejaba de cuestionarse si sería capaz de soportar dicha manifestaciones de apremio.

Finalmente, el tan esperado informe de los inspectores, no hizo otra cosa que fortalecer aún más, si cabe, la postura de la Casa Blanca y en este sentido evidentes son las declaraciones de su Secretario de Estado, Collin Powell, quien consideró dicho informe como: "un catálogo de no cooperación iraquí e insistió que la posición estratégica de Irak engaña a los inspectores" (La Nación, 8 de marzo, 2003, p.12).

Por su parte, Alvear reiteró ante el Consejo de Seguridad la vocación de paz de Chile además de la necesidad de alcanzar un desarme inmediato, cabal y efectivo en Irak. De igual manera, enfatizó que la vía diplomática era todavía viable: “...una solución que compatibilice el anhelo de paz es aún posible" (El Mercurio, 8 de marzo, 2003, p.A-4). No obstante, si bien el propio Lagos y sus personeros diplomáticos sabían que ese tiempo por el cual habían abogado se estaba agotando, también se agudizaba una situación que día a día se acrecentaba y que la propia Canciller Alvear no dejó pasar, de allí que expresara críticas directas al Gobierno iraquí en términos que Hussein estaba exponiendo a su pueblo a duros padecimientos.

Este último hecho, resulta gravitante para comprender otra arista no sólo de la conformación de la política exterior de Lagos, sino lo que ha caracterizado también al país, en el sentido de abogar constantemente por la población civil, que resultaba ser la gran y única víctima de

9. El 8 de noviembre de 1995, durante el 50o periodo ordinario de sesiones de la Asamblea General se favoreció en primera vuelta a Chile con la más alta votación de la Asamblea: 168 votos de un total de 177 efectivamente emitidos. Memoria del Ministerio de Relaciones Exteriores de Chile 1995, capítulo II, Asuntos Multilaterales, punto a, Naciones Unidas, 1) elección de Chile al Consejo de seguridad, p.142. 
los diferendos en torno a las supuestas armas de destrucción masiva. De allí que el gobierno chileno apeló por introducir ajustes legales al régimen de distribución de ayuda humanitaria al amparo del programa Petróleo por Alimentos ${ }^{10}$, ya que éste se había visto empañado por diversas acusaciones de mala gestión

Ante la temática relativa a la población civil, cabe señalar un punto importante que ha guiado la participación chilena al interior de Naciones Unidas, y que en el bienio 2003-2004 no fue la excepción: los Derechos Humanos. Por cierto, un punto muy sensible para Chile, no sólo en su etapa reciente; pensemos que uno de los redactores de la Declaración Universal de 1948, fue el chileno Hernán Santa Cruz Barceló, quien además lideró el Consejo Económico y Social (ECOSOC) y el equipo diplomático durante el primer bienio de Chile al interior del Consejo de Seguridad: "un imperturbable hombre de izquierda que ayudó a garantizar que los derechos económicos y sociales tuvieran un destacado lugar en la Declaración junto con las tradicionales libertades civiles y políticas" (Glendon, 2011, p.32)

Por lo tanto, se establece que la participación diplomática chilena a través del tiempo en relación a este organismo, ha estado marcada por un denominador común: una concepción humanitaria, democrática, idealista y jurídica:

el respeto al derecho internacional, a la ley y a los tratados; la presencia permanente de sus diplomáticos en los diversos esfuerzos de cooperación política latinoamericana y la participación de Chile como mediador en algunos conflictos, van a afianzar esa concepción democráticas -idealista y juridicista- de las relaciones internacionales. Estas fuentes son las que nutren el activismo internacional, en la que se distinguen algunos intelectuales y diplomáticos (Bernal-Meza, 2013, p.68).

\section{EN EL OCASO DEL TIEMPO: UNA PROPUESTA SE HACE PRESENTE}

Nos preguntábamos en la introducción del presente artículo, qué principios guiaron la política exterior chilena y cómo el papel que desarrolló la Delegación no Permanente, vinieron a graficar el accionar de Chile como un actor dentro del concierto internacional.

Al respecto, y siempre en el contexto de no llegar a la intervención directa en Irak, el gobierno presentó su propia fórmula para generar un consenso internacional, el desarme iraquí por medios

10. Oficio №675, Ref.: Proyecto resolución, Ministerio de Relaciones Exteriores, Gobierno de Chile Misión Chile-ONU, 20 de marzo, 2003. Asimismo, el Consejo de Seguridad solicitó al Secretario general de ONU que informara a éste cada seis meses sobre la operación de UNAMI (Misión de Asistencia de Naciones Unidas). Oficio № 158, Ref.: Proyecto resolución reconstrucción de Irak, reino Unido, Ministerio de Relaciones Exteriores, Gobierno de Chile Misión Chile-ONU, 1 de abril, 2003. 
pacíficos evitando así una crisis mayor al interior no sólo del propio Consejo de Seguridad, sino que también en la Asamblea General: "La propuesta de chile había sido formulada de buena fe y no como táctica dilatoria" (Muñoz, 2005: 73). Aunque debemos reconocer que si bien es cierto a esta altura, la crisis era latente, aún quedaban ciertas instancias para aminorar el costo que ésta podría tener y Chile al parecer lo tenía claro.

Los puntos centrales de la propuesta consideraron 5 ejes axiales: a) el régimen de Hussein debía en 3 semanas, poner a disposición del organismo competente a 30 científicos que podían ser entrevistados fuera del país, b) poner al descubierto todo proyectil de gas mostaza y exhibir todo documento que comprobara su destrucción, c) poner al descubierto 10 mil litros de ántrax o documentos que evidenciaran su destrucción, d) destruir todos los misiles Al Samud Il y sus componentes, e) hacer pública la información sobre los vehículos de control remoto y, por último, f) demostrar que no eran portadores de armas químicas (La Nación,15 de marzo, 2003, p.10).

Para algunos sectores, dicha iniciativa llegaba en un momento crucial, ya que se vislumbraba una tenue luz de paz producto de la aparente cooperación que había comenzado a mostrar Irak al iniciar el proceso de destrucción de los misiles Al Samud II; no obstante: "Estados Unidos rechazó el borrador de Chile. De acuerdo al vocero de la Casa Blanca Ari Fleisher la propuesta...-era un non starten". (Muñoz, 2005, p.72).Pese a haber encontrado una buena recepción entre los demás miembros del Consejo de Seguridad.

Al respecto, si bien el Gobierno chileno entendía la postura de EE. UU. en el sentido de ver amenazada la seguridad mundial, insistía en sus declaraciones públicas que la paz era posible siempre y cuando se fijaran metas verificables y plazos prudentes. Ahora bien, la búsqueda de un camino propio en pos de una vía que no significara un alto costo humanitario, logró al interior de Chile un momento pocas veces visto desde el regreso a la democracia en 1990, en términos de la unidad de todas las bancadas parlamentarias y sectores tanto políticos como sociales (Fuentes, 2010).

Hecho ratificado en reunión del 13 de marzo de 2003 en el palacio de La Moneda, ocasión en que estos sectores manifestaron respaldo absoluto al accionar del mandatario chileno en no dejarse presionar, a pesar de las consecuencias que esta decisión podría traer en torno a las negociaciones del TLC con EE. UU. (La Nación, 15 de marzo, 2003, p.10).

A pesar de las intenciones de aportar y generar una vía alterna que pavimentara un camino hacia acuerdos favorables, el tiempo y los intereses no entraron un punto en común. Así las cosas, a la semana siguiente de que se presentara la propuesta chilena, la prensa del periodo recogió ampliamente el mea culpa de Lagos y el llamado de atención que realizó al Consejo de Seguridad ante el fracaso de las gestiones diplomáticas para detener la guerra en el Golfo Pérsico: "entre los quince miembros del Consejo de Seguridad no fuimos capaces de encontrar una solución y por lo tanto debemos lamentar aquello" (El Mercurio, 19 de marzo, 2003, p.C-5). 
Evidentemente que la personalidad y el temperamento fuerte y decidido del Presidente Lagos se hizo notar en sus declaraciones ante la prensa, puesto que para él, el que Chile fuera parte del Consejo de Seguridad revestía una responsabilidad y un carácter trascendente en su visión de política exterior, en el entendido de posicionar a Chile en las altas cumbres internacionales. Pero esto no iba aislado, sino que también llevaba inserto una responsabilidad que ya indicábamos anteriormente en orden a la defensa de los derechos humanos, consensos y multilateralismos ${ }^{11}$.

Ahora bien, a pesar de la negativa a la propuesta presentada, todo este clima de incertidumbres y presiones diversas, le significó a Chile un reconocimiento de sus pares, ante una problemática que no sólo estaba ocurriendo a miles de kilómetros como era el caso en Irak, sino que también, como lo hemos mencionado ya, en el propio seno del Consejo de Seguridad en Nueva York ${ }^{12}$. Al respecto, consideramos importante destacar la valoración pública que realizó el Primer Ministro del Reino Unido, Tony Blair, ante la Cámara de los Comunes en torno a la iniciativa chilena de paz: "Estuvimos muy cerca de tener un acuerdo mayoritario y agradezco particularmente al Presidente de Chile por la constructiva forma en que abordó el asunto" (EI Mercurio, 20 de marzo, 2003, p.C-5).

En el Palacio de la Moneda se recibió con gran satisfacción las palabras de apoyo y reconocimiento del líder del Reino Unido. Pero no sólo los voceros del Gobierno expresaron su sentir respecto a las palabras de Blair, sino que el mismo Ricardo Lagos indicó a la prensa chilena el agradecimiento al Primer Ministro británico. En esta misma instancia de mutuos reconocimientos, el presidente Lagos no dejó pasar la oportunidad de nuevamente dirigir un mensaje no tan sólo a los chilenos, sino al mundo entero ante el fracaso diplomático: "Siento un lamento por el mundo, porque hoy creo que el mundo tiene preocupación por lo que va a ocurrir, tiene ansiedad por lo que pasará y esperemos que los sufrimientos sean mínimos" (El Mercurio, 19 de marzo, 2003, p.C-5).

Palabras que quedarán sin lugar a dudas en los anales de la historia reciente chilena, puesto que a esas alturas se tenía absoluta claridad de lo que sucedería unas horas más tarde, cuando se llevó a cabo el abandono del multilateralismo y quiebre transatlántico, aquel 19 de marzo de 2003: "Apenas habían pasado unas pocas horas desde la reunión del Consejo de Seguridad cuando, a las 21.35 hrs., hora de la costa este de Estados Unidos, cayeron las primeras bombas norteamericanas sobre Bagdad" (Muñoz, 2005: 93). Tras lo cual el Presidente Bush se dirigió a las cámaras de televisión para expresar: "No esperamos otro resultado que la victoria" (El Mundo, 2003).

11. Oficio № 923, Ref.: Proyecto discurso Presidente Lagos en Asamblea, Ministerio de Relaciones Exteriores, Gobierno de Chile, Dirección de Comunicaciones, junio de 2004

12. Oficio №136, Ref.: Intervención de Chile en el debata sobre Irak en el Consejo de Seguridad, Ministerio de Relaciones Exteriores, Gobierno de Chile Misión Chile-ONU, 19 de marzo, 2003. 
Tras la iniciativa del gobierno y su equipo diplomático, ¿qué logró en concreto Chile?, sin lugar a dudas, fortalecer la imagen país, de pequeña nación pero con aspiraciones mayores. Un ideal marcado por una opción que no recibió el respaldo esperado y que guardaba una preocupante singularidad, en orden a lo suponía colocarse en una estrecha cornisa junto a EE. UU. A la vez que, por su parte, los informes del equipo de inspectores de la ONU, vinieron a apoyar los lineamientos planteados por EE. UU. y Reino Unido: "This behavior cleared the road for Bush and Blair to launch a preemtive invasion ostensibly to prevent the use of those presured weapons, even with-out U.N. support" (Murray y Scales, 2003, p.42).

Cuando se analizan las declaraciones de los líderes chilenos, es posible vislumbrar preocupación por la tensión existente, pero a la vez, confianza de que finalmente se lograría separar las relaciones bilaterales entre ambos países de lo que estaba ocurriendo al interior de Naciones Unidas, com lo que sucedió el 6 de junio del año 2003, cuando se firmó entre ambos países el tan anhelado Tratado de Libre Comercio, TLC (Marisio, 2004).

\section{CONCLUSIÓN}

Apenas iniciándose el siglo XXI el mundo fue testigo del primer gran conflicto a escala, que no sólo afectó a aquellos países directamente relacionados con la invasión a Irak, sino que supuso la participación de aquellas naciones que para el periodo eran parte del Consejo de Seguridad. En este sentido, el conflicto iraquí significó para Chile una oportunidad de desarrollar un papel activo, quizá más que nunca, en comparación con los otros tres bienios en que fue parte de dicho Consejo.

Asimismo, esta ocasión le permitió a Chile a pesar de las diferencias internas, conducirse políticamente bajo el alero de decisiones consensuadas entre los diversos sectores, a pesar que una sombra de temor se hacía presente, vinculada directamente con la firma del TLC con EE. UU. Aunque también, se debía considerar los efectos económicos que produciría dicha intervención sobre el precio fijo del petróleo.

Al inicio de este trabajo, surgía el interrogante ¿cuál fue la lección que aprendió Chile de este conflicto? Ante ello, podemos sostener que entendió que no debía precipitarse en la toma de decisiones ni mucho menos llegar a algún tipo de acuerdo sin antes tener sobre la mesa todos los antecedentes que pudieran entregar el equipo de inspectores observadores en Irak. Porque aquello del tiempo, resultó ser el objetivo central que defendió el Gobierno del presidente Lagos a pesar de las presiones constantes que recibió para dar su voto a favor de la invasión.

De igual manera, el gobierno chileno y su equipo diplomático, a pesar de las constantes críticas hacia Naciones Unidas, favoreció el revitalizar al propio Consejo de Seguridad por medio de la cooperación internacional. Una actitud que visualizó en la multilateralidad un 
camino para llegar a convertirse en aquella piedra angular sobre la cual debiera descansar la esperanza de poder lograr una paz estable, desterrando por esta vía el cáncer que significa el terrorismo.

En ello también la prensa desempeñó un rol preponderante, en orden a ser el nexo entre los centros de poder y la opinión pública, ya que ésta se transformó en una verdadera protagonista de la diplomacia, en términos de la forma de representación del hecho y las presiones que a su vez pudo hacer.

El camino que emprendió Chile ante el desafío de ocupar un escaño en el Consejo de Seguridad para el año 2003, le significó comprender y poner el acento en que ninguna nación por sí sola, independiente de lo poderosa que fuera, puede desarrollar una acción unilateral en un escenario complejo sin el apoyo y la real participación de las demás naciones.

Esto fue lo que el Presidente Bush junto al resto de países que los apoyaron, pudieron observar luego de lanzarse en una guerra contra el régimen de Hussein, bajo unas justificaciones que no estuvieron comprobadas por los organismos internacionales como la UNMOVIC, ni respaldada por todos los miembros del Consejo de Seguridad.

En definitiva y de acuerdo a lo que se pretendió subrayar en este trabajo, podemos sostener que Chile como Miembro no Permanente del Consejo de Seguridad comprendió que debía responder a la confianza que se le había depositado para participar en la estabilidad y la seguridad internacional. Para ello, se apegó a los lineamientos del derecho internacional y a lo que establecía la Carta constitutiva de Naciones Unidas, potenciando un multilateralismo en vez de la unilateralidad.

De allí y a pesar de los cuestionamientos que generó y sigue generando la funcionalidad real de Naciones Unidas, tenemos que entender que un organismo de este tipo resulta ser clave para el desarrollo en conjunto de los pueblos, por lo tanto aún queda mucho por hacer.

Finalmente, también debemos indicar que para abordar el estudio de la participación de Chile al interior del Consejo de Seguridad de Naciones Unidas, un gran obstáculo resultó ser la ausencia bibliográfica frente a esta temática. Puesto que la gran mayoría de los estudios existentes abarcan perspectivas desde del derecho internacional, quedando un gran desafío para los historiadores, quienes desde la perspectiva propia de la disciplina pueden contribuir a la historia del tiempo reciente de Chile, en el ámbito de la historia de las relaciones internacionales.

\section{REFERENCIAS}

Agüero, F. (2005). "El acuerdo de libre comercio Chile-Estados Unidos", Colombia Internacional, №61, enero-junio, pp.50-62. 
Arístegui, G. (2003). "¿Tercera Guerra del Golfo?”, Política Exterior, №92, marzo-abril, pp.99-111.

Bernal-Meza, R. (2013). América Latina en el mundo. Buenos Aires: Grupo Editor Latinoamericano.

El Mercurio (2003, 17 de enero), Expectación causa 12 ojivas encontradas en Irak. Edición impresa, p.A-4.

(2003, 28 de febrero). Fuerte crítica chilena a potencias de la ONU, Edición impresa, p.A-1.

(2003, 8 de marzo). Reafirmamos la necesidad de un desarme inmediato, cabal y efectivo. Edición impresa, p.A-4.

(2003, 19 de marzo). Lagos asume cuota de responsabilidad en fracaso de gestiones de la ONU. Edición Impresa, p.C-5

El Mundo (2003, 20 de marzo). Guerra en Irak, Recuperado de http://www.elmundo.es/ especiales/2003/02/internacional/irak/ardebagdad.html. Consultado el 20.9.2014

Fazio, H. (2004). TLC, el amarre del modelo. Santiago de Chile: LOM Ediciones.

Fermandois, J. (2004). Mundo y fin de mundo. Chile en la política mundial 1900-2004. Santiago de Chile: Ediciones Universidad Católica de Chile.

Fuentes, C. (2010). "Progresismo y política exterior: el caso de Chile". En Quiroga, Y. (Ed.), Consenso progresista. Política exterior de los gobiernos progresistas del Cono Sur: convergencias y desafío, Santiago de Chile: Friedrich Ebert.

Gomis, L. (1991). Teoría del periodismo. Cómo se forma el presente. Barcelona: Paidós.

Glendon, M.A. (2011). Un muerdo nuevo. México: Fondo de Cultura Económica.

La Nación (2003, 17 de enero). Hallan ojivas químicas vacías en Irak. Edición impresa, p.14.

(2003, 21 de enero). ONU e Irak hicieron las paces. Edición impresa, p.14.

(2003, 23 de enero). EE.UU. tiene fecha para atacar Irak. Edición Impresa, p.15.

(2003, 29 de enero). Irak: ONU lucha contra el tiempo. Edición impresa, p.15.

(2003,7 de marzo). Espionaje a Chile en la ONU. Edición impresa, p.12. 
(2003, 15 de marzo). La propuesta chilena. Edición impresa, p.10

Llanos, H. (1996). "Cumplimiento en Chile de las decisiones del Consejo de Seguridad de las Naciones Unidas", Revista Chilena de Derecho, Vol. 23, № 2, Tomo I, pp.309-340.

Marisio, A. (2004). “Una crónica de la aprobación del TLC” Diplomacia № 100, pp.120-145.

Mensaje Oficial 014, REF: Posición EE.UU. respecto resolución sobre Irak, Ministerio de Relaciones Exteriores, Gobierno de Chile, Dirección de Política Multilateral, Departamento de Naciones Unidas 22 enero, 2003.

Mensaje Oficial № 026, Ministerio de Relaciones Exteriores, Gobierno de Chile, Dirección de Política Bilateral, 4 de febrero de 2003.

Memoria del Ministerio de Relaciones Exteriores de Chile 1995, capítulo II, Asuntos Multilaterales, punto a, Naciones Unidas, 1) elección de Chile al Consejo de seguridad, p.142

Milet, P. (2004). Más voces para la democracia. Los desafío de la sociedad civil. Santiago de Chile: Más Voces.

Mintz, Alex y Karl De Rouen Jr., (2010), Understanding foreign policy decision making, Nueva York: Cambridge University Press.

Muñoz. H. (2005). Una guerra solitaria. Santiago de Chile: Random House Mondadori S.A.

Murray.W y Scales, R (2003). The Iraq war. An elusive victory. United States of America: Harvard University.

Nicolson, H. (2010). La Diplomacia, México: Fondo de Cultura Económica.

Oficio № 100, Ministerio de Relaciones Exteriores, Gobierno de Chile, Misión Chile-ONU, 15 de septiembre, 2002

Oficio № 097, Ref: Proyecto resolución Sirio Consejo de Seguridad, Ministerio de relaciones Exteriores, Gobierno de Chile, Misión Chile-ONU, 17 de abril, 2003.

Oficio №075, Ref: Proyecto resolución, Ministerio de Relaciones Exteriores, Gobierno de Chile Misión Chile-ONU, 20 de marzo, 2003.

Oficio № 158, Ref: Proyecto resolución reconstrucción de Irak, reino Unido, Ministerio de Relaciones Exteriores, Gobierno de Chile Misión Chile-ONU, 1º de abril, 2003. 
Oficio № 923, Ref: Proyecto discurso Presidente Lagos en Asamblea, Ministerio de Relaciones Exteriores, Gobierno de Chile, Dirección de Comunicaciones, junio de 2004

Oficio №136, Ref: Intervención de Chile en el debata sobre Irak en el Consejo de Seguridad, Ministerio de Relaciones Exteriores, Gobierno de Chile Misión Chile-ONU, 19 de marzo, 2003.

Palomares, G., García, P. (1999). Relaciones Internacionales. Sociedad internacional. Madrid: Addenda, Universidad Nacional de Educación a Distancia.

Pereira, J.C. y Martínez Lillo, P. (2001). La ONU. Madrid: Arco Libros.

Pérez, C. (1998). El regreso de la historia. La política internacional durante la posguerra fría: 1989-1997. Buenos Aires: Editorial Sudamericana.

Publimetro, (24 de septiembre de 2013). Piñera: "Chile va muy bien encaminado al Consejo de seguridad de la ONU". Recuperado de: http://www.publimetro.cl/nota/ politico/pinera-chile-va-muy-bien-encaminado-al-consejo-de-seguridad-de-la-onu/ xIQmix!ICsLVXgksboGM/. Consultado el 18.9.2014.

Quezada, A. (2010). "Inserción internacional de Chile en la post-Guerra Fría. Concertación política e integración económica comercial; dos ejes conceptuales de la política exterior en el Gobierno de Ricardo Lagos 2000-2006". Enfoques, Vol. VIII, № 13, pp. 119-134.

Remiro, A. (2003). "La tentación de la agresión. Ilegalidad del unilateralismo". Política Exterior, №92, (marzo-abril), pp.19-23.

Talmon, S. (2007). "A plurality of responsable actors: internacional responsibility for acts of the coalition provincial authority in Iraq". Londres, University of Orford, Faculty of Law Legal Studies Research, Papers Series, Working Papers №25/2007, september, pp.1-42.

Ulloa, E. (2013). Chile ante el Consejo de Seguridad de Naciones Unidas. Acción diplomática y opinión pública 1952-2004. (tesis de grado para optar al título de Doctor en Historia Contemporánea). Valladolid, España: Universidad de Valladolid.

Ulloa, E. (2013). "Chile y el Consejo de Seguridad". El Mercurio, 22 de octubre, p.A-2.

Vargo, R. (2004). "La negociación del Acuerdo de Libre Comercio EE.UU.-Chile", Diplomacia № 100, pp. 72-78.

Veiga, F. (2009). El desequilibrio como orden. Una historia de las Posguerra Fría 1990-2008. Madrid: Alianza Editorial. 